\title{
An exposure effect opposes flavor-nutrient learning
}

\author{
JACQUELINE OWENS, ELIZABETH D. CAPALDI, and JOAN D. SHEFFER \\ University of Florida, Gainesville, Florida
}

\begin{abstract}
When a caloric substance is followed by a flavored substance, preference for the flavor that followed the calories can increase because of a flavor-nutrient association. We showed here that this effect is opposed by a fullness effect: consuming the caloric substance itself reduces consumption of the flavor that follows. Because consumption of this flavor was less than consumption of a flavor that was given alone, there was a reduced preference for the flavor that followed the calories-an effect opposing flavor-nutrient learning. The preference for the flavor that was given alone and consumed in greater amounts seems to be due to exposure per se and is not a result of contrast. When the amount of the flavor given alone was directly controlled so that it was one half the amount of the flavor that followed the calories, preference for the flavor following the calories was no higher than when consumption of the two flavors was equal. Thus, exposure can interfere with flavor-nutrient learning, but does not enhance it.
\end{abstract}

Capaldi and Sheffer (1992) showed that a flavor of saccharin given after chocolate milk was later preferred to a flavor given alone. They suggested that the flavor given after the chocolate milk was associated with the postingestive effects (nutrients) of the chocolate milk, and was therefore later preferred on the basis of flavor-nutrient learning. Boakes and Lubart (1988) suggested this hypothesis to account for their finding that a flavor of saccharin given shortly after glucose was preferred to a flavor given alone. When flavored saccharin follows glucose, the flavor of saccharin is more closely associated in time with the postingestive effects of glucose than is the flavor of glucose itself. Consistent with this hypothesis, Boakes and Lubart (1988) showed that a flavor of saccharin given $60 \mathrm{~min}$ after glucose, long enough for the postingestive effects of glucose to have dissipated, was not preferred to a flavor given alone.

In Experiment 1, we were concerned with whether exposure to flavored saccharin or the nutrient prior to flavor-nutrient learning would interfere with that learning. Holder (1991) showed that a cue (taste or odor) that had been paired with sucrose was preferred to one that had been paired with saccharin. When a second cue was added that was associated with sucrose, the second cue was ignored in favor of the cue that was learned first-a blocking effect (Kamin, 1969). We hypothesized that as a result of preexposure to chocolate milk, rats would form an association between the taste of chocolate milk and the resulting calories, and that when saccharin followed chocolate milk in training, the saccharin cue would be ig-

This research was supported in part by Grant MH39253 to the second author from the National Institute of Mental Health. Requests for reprints should be sent to E. D. Capaldi. Department of Psychology, University of Florida, Gainesville, FL 32611-2065. nored. Similarly, we predicted that a group preexposed to a flavor of saccharin would learn that the flavor had no caloric consequences, and when this flavor followed chocolate milk, the flavor would be less likely to be associated with the postingestive effects of chocolate milk than the chocolate milk itself. Both of these effects would interfere with forming an association between the calories from chocolate milk and the flavor of saccharin that followed. It was predicted that a group having no preexposure would show a preference for the flavor of saccharin that followed chocolate milk because no blocking would occur. The results of Experiment 1 indicated that there is a factor interfering with flavor-nutrient learning; however, the nature of that interference was not as we predicted. The subsequent two experiments were conducted to clarify the characteristics of this interfering factor and to determine to what degree it affects flavor- nutrient learning.

\section{EXPERIMENT 1}

In the Experiment 1 training phase, all the rats received chocolate milk followed by saccharin Flavor 1 on 4 days and only saccharin Flavor 2 on 4 days. It was predicted that this training would produce a preference for Flavor 1 that was based on flavor-nutrient learning (Boakes \& Lubart, 1988; Capaldi \& Sheffer, 1992). Prior to this training, we preexposed different groups to saccharin Flavor 1, saccharin Flavor 2, chocolate milk, or nothing. We reasoned that preexposure to saccharin Flavor 1 or chocolate milk might interfere with flavor- nutrient learning when Flavor 1 followed chocolate milk in training. Preexposure to saccharin Flavor 1 might teach the rats that the flavor contained no nutrients, whereas preexposure to chocolate milk might teach them that the flavor of chocolate milk precedes its postingestive effects. Either of these associations could interfere with the rats' learning that saccha- 
rin Flavor 1 is associated with the postingestive effects of chocolate milk.

\section{Method}

Subjects. The subjects were 40 naive Sprague-Dawley rats from Harlan Co., Indianapolis, IN. They were 75 days old upon arrival and 78 days old at the start of the experiment.

Solutions. The solutions were presented in $50-\mathrm{ml}$ Nalgene centrifuge tubes with rubber stoppers and metal spouts. They were flavored with $.15 \%$ saccharin and Gustafson's chocolate milk. The flavorings consisted of $.05 \%$ grape and $.05 \%$ cherry unsweetened Kool-aid powder (General Foods).

Procedure. Water was available ad lib throughout the experiment in clear plastic bottles mounted to the right of the food hopper on the cage front. On the first day of the experiment, all food was removed from the cages and for the next 10 days the rats were fed $14 \mathrm{~g}$ of lab chow at what would be their training time. They were kept on this deprivation schedule for the remainder of the experiment, except that during training and testing they were fed $2 \mathrm{~h}$ after the sessions were run. The calories consumed via the chocolate milk were accounted for and rations were reduced so that the caloric equivalent of $14 \mathrm{~g}$ was given to each rat daily.

There were 8 preexposure days, 8 training days, and 4 test days of saccharin Flavor 1 versus saccharin Flavor 2.

In preexposure, Group Flavor1 was given the flavor of saccharin that would follow chocolate milk in training (Flavor 1); Group Flavor2 was given the flavor of saccharin that would be given alone in training (Flavor 2); Group Chocolate was given chocolate milk; and the control group received no solutions during this phase. All the solutions were given once a day in $40-\mathrm{ml}$ portions for $10 \mathrm{~min}$.

After the preexposure phase, the training procedure was the same for all the groups. On half the days, according to a double alternation schedule, each rat received one tube of chocolate milk for $10 \mathrm{~min}$, followed $5 \mathrm{~min}$ later by a tube of saccharin Flavor 1. On the remaining days, the rats received only Flavor 2 for $10 \mathrm{~min}$. Flavor was counterbalanced so that Flavor 1 was cherry and Flavor 2 was grape for half the rats in each group; for the remaining rats, Flavor 1 was grape and Flavor 2 was cherry. On Day 1 , half the rats in each group received chocolate milk followed by saccharin Flavor 1 and the other half received only saccharin Flavor 2 . Consumption was recorded.

In test, all the rats had a choice of either $40 \mathrm{ml}$ of grape- or cherryflavored saccharin. A tube was inserted and moved back and forth until the rat licked the tube end; then the tube was removed and the other tube was inserted and moved back and forth until the rat tasted this solution. Then both tubes were left on the cage front for $10 \mathrm{~min}$. Position was alternated so that grape was on the left on the first and last test days, and cherry was on the left on the second and third days. Consumption was recorded.

\section{Results}

Saccharin consumption during training (mean milliliters consumed over days by each group) is shown by group in Figure 1. As can be seen, all the groups in training drank more of the flavor of saccharin that occurred alone (Flavor 2) than the flavor that followed chocolate milk (Flavor 1), and the groups preexposed to saccharin drank more saccharin than the other two groups. An analysis of variance (ANOVA) showed that the difference due to Flavor 1 versus Flavor 2 was significant $[F(1,36)=$ $104.79, p<.01]$, as was the difference associated with groups $[F(3,36)=25.71, p<.01]$ and the group $\times$ flavor interaction $[F(3,36)=13.85, p<.01]$. Subsequent Newman-Keuls tests $(p<.05)$ showed that the groups

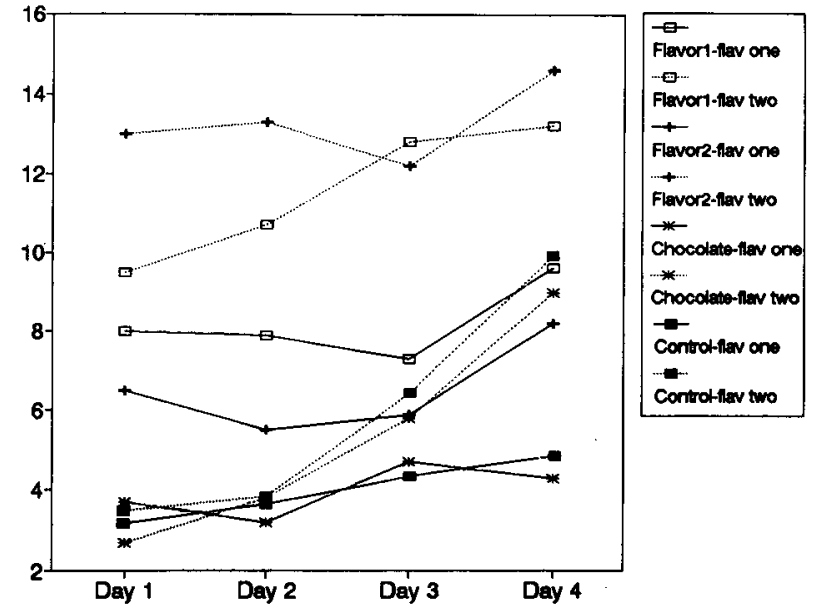

Figure 1. Mean consumption (in milliliters) of the flavor saccharin that followed chocolate milk (Flavor 1) and the flavor that occurred alone (Flavor 2) for each group over training days in Experiment 1.

preexposed to saccharin drank more of Flavor 2 than of Flavor 1, whereas the other two groups' consumption of Flavors 1 and 2 did not differ significantly. These tests also showed that the groups preexposed to saccharin drank more of Flavors 1 and 2 than did the other two groups.

Chocolate milk consumption in training (average consumption in milliliters) for Group Flavor 1 was 12.5, for Group Flavor2 was 12.1, for Group Chocolate was 17.5, and for Group Control was 12.8. An ANOVA showed that the group difference was significant $[F(3,36)=$ $23.47, p<.01]$.

Figure 2 shows the consumption of the two flavors of saccharin in test for each group. Although all the groups except Group Chocolate had a consumption difference favoring the flavor of saccharin that occurred alone to the flavor that followed chocolate milk, none of the differences were significant, including that associated with flavor $[F(1,36)=2.98, p=.0931]$.

\section{Discussion}

In training, the groups drank more of the solution that they were preexposed to (saccharin or chocolate milk) than did the groups not preexposed to that solution. This result is compatible with other findings, such as Domjan's (1976) results, which showed that exposure to saccharin increases consumption of saccharin-a phenomenon that has been attributed to "learned safety." As a result of experiencing the novel taste of saccharin with no negative consequences, neophobia is reduced and saccharin consumption increases. Exposure to either flavor of saccharin increased consumption of both, whereas exposure to chocolate milk did not.

It is interesting to note in Figure 1 the pattern of saccharin consumption for the groups preexposed to saccharin. In the first days of training, when the effects of preexposure should be strongest, Group Flavorl would be expected to consume more of saccharin Flavor 1, because this is the 


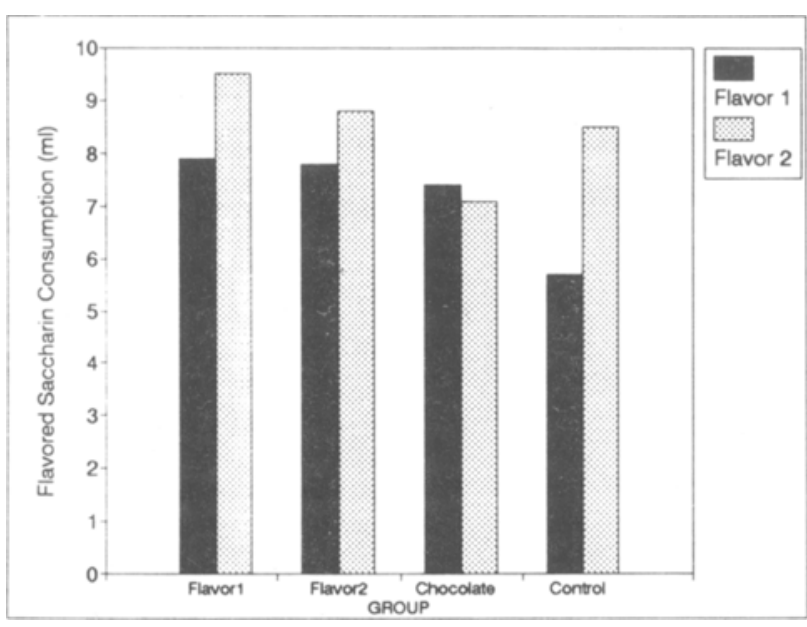

Figure 2. Mean consumption (in milliliters) of the flavor saccharin that followed chocolate milk (Flavor 1) and the flavor that occurred akone (Flavor 2) for each group averaged over testing in Experiment 1.

flavor they were preexposed to. However, this did not occur. Although they consumed similar amounts of the two flavors at the beginning of training, they never drank more of saccharin Flavor 1 than Flavor 2. Group Flavor2, on the other hand, drank much more of saccharin Flavor 2 than Flavor 1, with the difference evident early in training and lasting throughout training. Possible explanations for this suppressed consumption of Flavor 1 by both groups will be discussed after presentation of Experiment 2 .

Contrary to previous results, the rats did not prefer the flavor of saccharin that followed chocolate milk. We expected that exposure to Flavor 1 or chocolate milk would interfere with flavor-nutrient learning, but none of the groups showed a preference for the flavor following chocolate milk, not even the control group that received no preexposure. Preference was in the other direction for all the groups, except Group Chocolate. This is inconsistent with the results of Capaldi and Sheffer (1992), who found a preference for a flavor that followed chocolate milk over a flavor given alone; that is, flavor-nutrient learning.

In Experiment 1 training, all groups consurned less of the flavored saccharin that followed chocolate milk than of the flavor that occurred alone. This may indicate a negative contrast effect. Saccharin is less of a reward than chocolate milk, and consumption of a lesser reward is suppressed when it occurs in the context of a more preferred reward (Flaherty, 1982).

Another explanation is that the rats may have consumed less of the flavor of saccharin that followed the chocolate milk because of a temporary satiation effect that resulted from consuming chocolate milk 5 min earlier. The feeling of fullness that would result from consuming the chocolate milk may have suppressed consumption of the saccharin that followed; on the days in which saccharin was the only solution given, no such fullness factor inhibited drinking. There may be a reduced preference for the flavor whose consumption is reduced because there is less consumption of that taste, and therefore less habituation of neophobia for that taste.

In Experiment 2, we allowed negative contrast to operate, but we controlled for fullness to determine whether contrast was affecting flavor-preference learning.

\section{EXPERIMENT 2}

In Experiment 2, the rats received saccharin Flavor 1 following chocolate milk and saccharin Flavor 2 following white milk. This method was used in order to equate any fullness effect, because both flavors of saccharin followed a flavor of milk. If fullness opposed flavor-nutrient learning in Experiment 1, controlling for it here should allow expression of flavor-nutrient learning. Specifically, the rats should prefer the flavor following the more caloric chocolate milk. If contrast effects oppose flavor- nutrient learning when flavored saccharin is given with milk, the rats should prefer the flavor following white milk. Because chocolate milk is preferred to white, there should be more of a negative contrast effect with chocolate milk and, therefore, less of a preference for the flavor following chocolate milk. Also, one group was preexposed to chocolate milk to determine what effect, if any, this would have on flavor-nutrient learning. In Experiment 1, although none of the groups had a significant preference for Flavor 1, the group preexposed to chocolate milk was the only group with consumption in test in the direction indicating flavor-nutrient learning.

\section{Method}

Subjects. The subjects were 20 rats of the same description as those of Experiment 1. They were 77 days old upon arrival and 84 days old at the start of the experiment.

Solutions. The solutions were the same as those used in Experiment 1, with the addition of Gustafson's whole white milk.

Procedure. Two groups of 10 rats, Groups Preexpose and No Preexpose, were run. The rats were fed, watered, and trained as in Experiment 1, with the following exceptions.

After the preexposure phase, which was conducted as in Experiment 1 ( 8 days of chocolate milk for Group Preexpose and no preexposure for Group No Preexpose), there were 8 days of training. All the rats received $40 \mathrm{ml}$ of milk for $10 \mathrm{~min}$, which was followed $5 \mathrm{~min}$ later by flavored saccharin for $10 \mathrm{~min}$ each day. The subjects received white milk on half the days and chocolate milk on the other days, according to a double alternation schedule. Flavor 1 of saccharin followed chocolate milk and Flavor 2 followed white.

Six test days followed this training. A 10-min, two-bottle test was run daily with $40-\mathrm{ml}$ of Flavors 1 and 2 , following the test procedure of Experiment 1. Two test days of chocolate versus white milk were run after the saccharin test phase, also following the test procedure of Experiment 1.

\section{Results}

Figure 3 shows milk consumption averaged over days in training for each group. As can be seen, the group preexposed to chocolate milk drank more of both milks than did Group No Preexpose, particularly more chocolate milk. An ANOVA conducted on milk consumption in training revealed a main effect of group $[F(1,18)=$ $42.21, p<.01]$, showing the greater consumption of milk by the preexposed group. There was also a main effect 


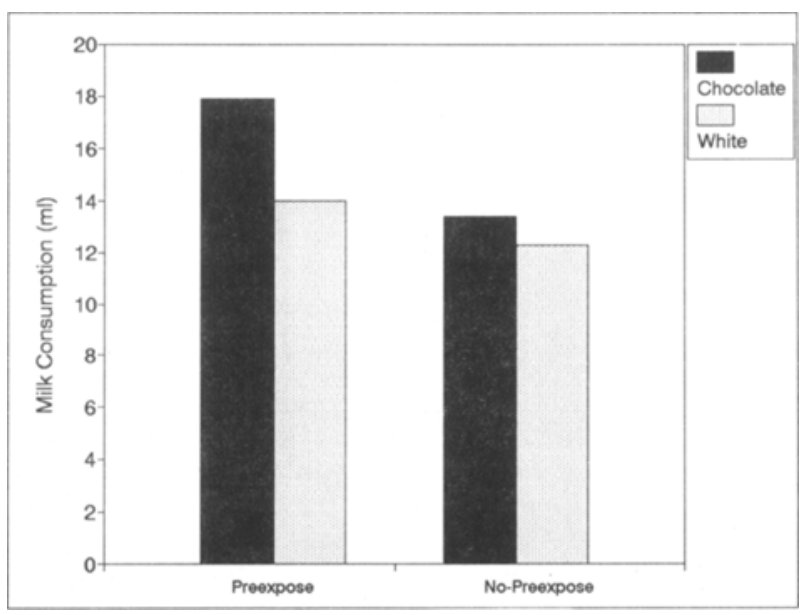

Figure 3. Mean consumption (in milliliters) of both chocolate and white milk for each group averaged over training in Experiment 2.

of milk type $[F(1,18)=66.65, p<.01]$, reflecting the greater consumption of chocolate milk by both groups. The group $\times$ milk type interaction was also significant $[F(1,18)=19.21, p<.01]$; subsequent Newman-Keuls tests $(p<.05)$ determined that Group Preexpose drank significantly more chocolate milk than white milk, whereas consumption of the two milks did not differ for Group No Preexpose.

There were no significant differences in saccharin consumption during training. Mean consumption of Flavors 1 and 2 averaged over training were 4.6 and $4.3 \mathrm{ml}$ for Group Preexpose, and 5.0 and $4.7 \mathrm{ml}$ for Group No Preexpose.

Average consumption of flavored saccharin over days by groups in test is shown in Figure 4 . In test, the animals preferred the flavor of saccharin that had followed chocolate milk (Flavor 1) to the flavor that had followed white milk (Flavor 2) [4.37 vs. $2.96 \mathrm{ml}, F(1,18)=$ $13.24, p<.01]$. This difference did not vary by group $[F(1,18)=1.21, p>.05]$.

In the second test (chocolate milk vs. white milk), both groups preferred chocolate milk to white $[F(1,18)=$ $34.81, p<.01]$, with an average consumption of $9.6 \mathrm{ml}$ of chocolate milk and $2.6 \mathrm{ml}$ of white milk. Preference for flavor of milk did not vary by group (milk type $x$ group interaction, $F<1.0$ ).

\section{Discussion}

In Experiment 2, we showed that flavor-nutrient conditioning does occur for a flavor that follows chocolate milk, whether there is preexposure to chocolate milk or not, if the influence of fullness is reduced. Negative contrast does not seem to be an important factor in blocking flavor-nutrient learning. Data from the second test confirmed that the chocolate milk was preferred to the white milk. Had negative contrast been a factor, the saccharin that followed chocolate milk would not have been preferred to the flavor that followed white milk. The rats nonetheless preferred the flavor that followed chocolate milk, showing that negative contrast did not block flavornutrient learning.

The fact that preexposure did not affect test preference in Experiment 2 points to a difference between conditioned aversions and preferences. Preexposure to a flavor can interfere with later attempts to condition illness to that flavor (Domjan, 1977). In Experiment 2, in which some groups were preexposed and some groups were not, the results did not vary by group, showing that preexposure does not affect flavor-nutrient learning.

One way that fullness may have controlled preference in test is by controlling exposure in training. In Experiment 1 , when the rats consumed the chocolate milk, the temporary fullness may have reduced the consumption of the saccharin Flavor 1 that followed the chocolate milk, whereas no such fullness inhibited the consumption of saccharin Flavor 2. This temporary fullness would explain why even Group Flavor 1, which had been preexposed to Flavor 1 and would be expected to consume more of that flavor because of a reduction in neophobia, drank more saccharin Flavor 2 in training. Group Flavor2, which had both preexposure and the fullness effect influencing consumption in the direction favoring Flavor 2, consistently drank more saccharin Flavor 2 throughout training. Saccharin Flavor 2, then, would be more familiar to the rats than saccharin Flavor 1. The failure to find a preference for Flavor 1 in testing, then, may be the result of more exposure to saccharin Flavor 2 than saccharin Flavor 1. The greater exposure to saccharin Flavor 2 may have interfered with the rats' showing a preference for Flavor 1.

In Experiment 2, fullness was equated over days by giving milk before both saccharin Flavors 1 and 2, thus reducing consumption of both flavors of saccharin in training. Comparing consumption of saccharin in the first two

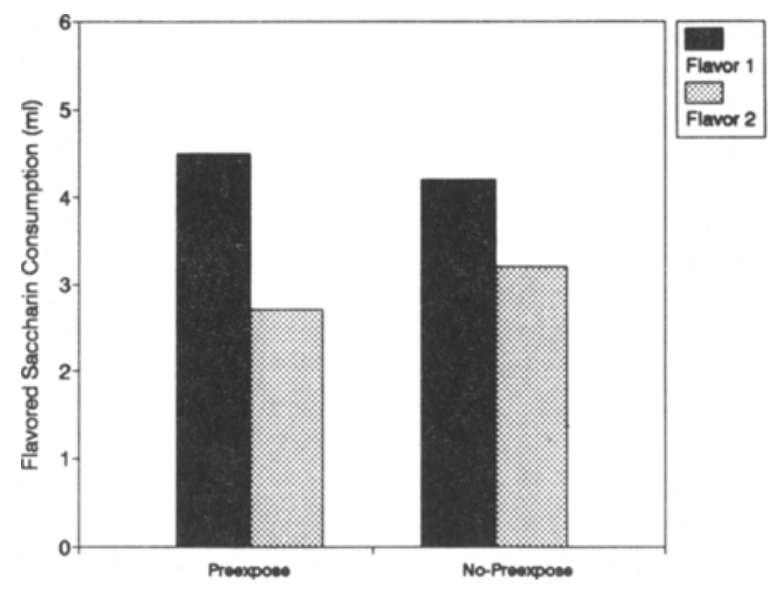

Figure 4. Mean consumption (in milliliters) of the flavor that followed chocolate milk (Flavor 1) and the flavor that followed white milk (Flavor 2) for each group averaged over testing in Experiment 2. 
experiments shows that the rats drank more saccharin in Experiment 1 than in Experiment 2 in both training and testing. Because of the limited experience with saccharin in training, neophobia to the saccharin was not reduced in Experiment 2 as much as it was in Experiment 1. This lower consumption of saccharin in training carried over into testing, where amounts consumed were again lower than in Experiment 1.

Another possibility is that fullness may produce a temporary negative state in the rat. After consuming the chocolate milk, the feeling of fullness may have been unpleasant; when the saccharin that followed the milk (Flavor 1) was consumed, the negative state may have been associated with that flavor of saccharin. The principles here are similar to those involved in flavor-nutrient learning. In flavor-nutrient learning, calories are associated with the flavor that is being consumed while the nutrients are being absorbed; here, the unpleasant state that would result from consumption of the first solution would be associated with the second solution. However, the fullness effect is not proposed as a caloric satiation; it is analogous to the state that follows consumption of a large amount of water. In this case as well, the fullness factor would work against flavor-nutrient learning, preventing a preference for the flavor that follows chocolate milk.

The procedure of Experiment 1 was followed in Experiment 3, but the amount of flavored saccharin consumed was directly manipulated to determine whether degree of preference for the flavor of saccharin following chocolate milk could be determined by differential exposure to the saccharin flavors.

\section{EXPERIMENT 3}

Two groups were run in Experiment 3. Both were given chocolate milk followed by Flavor 1 on the first day, and on alternate days thereafter. On the remaining days, only saccharin Flavor 2 was given. This was the same basic procedure as that used in Experiment 1, except that the amount of Flavor 2 was controlled. One group of rats was given an amount of saccharin Flavor 2 that was equal to the consumption of saccharin Flavor 1 on the previous day. The rats in the other group were given half as much saccharin Flavor 2 as had been consumed of Flavor 1 on the previous day so that, over training, these rats had more of the flavor of saccharin following chocolate milk. By eliminating the possibility that they would drink less of the saccharin that followed chocolate milk in training than of the flavor that occurred alone, we hoped to allow for flavor-nutrient learning to occur. According to our theory, both the groups should prefer the flavor of saccharin following chocolate milk. Also, if exposure is controlling degree of preference, we would expect the group that had twice as much of the flavor following chocolate milk to have a greater preference for that flavor than the group that had equal exposure to both flavors.

\section{Method}

Subjects. The subjects were 20 Sprague-Dawley rats from Charles River, Inc., Wilmington, MA. They were 77 days old upon arrival and 81 days old at the start of the study.

Solutions. The solutions were the same as those used in Experiment 1.

Procedure. There were two groups of 10 rats each. The rats were fed, watered, and trained as in Experiment 1, with the following exceptions.

There was no preexposure phase. The rats in both groups were given chocolate milk followed by saccharin Flavor 1 on half the days and saccharin Flavor 2 on half the days on a single alternation schedule. Chocolate milk and saccharin Flavor I were given to all the rats on the first day, and the amount consumed was recorded. On the next day, saccharin Flavor 2 was given to all the rats in the same amount that they had consumed of Flavor 1 the previous day. This procedure continued for 4 days.

On the fifth day, when consumption of saccharin was up to $2 \mathrm{ml}$ for every rat, consumption of saccharin Flavor 1 was measured. Then, on the sixth day, for Group Equal, saccharin Flavor 2 was given to each subject in an amount equal to the amount of saccharin Flavor 1 that was consumed by that subject on the previous day. For Group Half, saccharin Flavor 2 was given in half the amount that had been consumed of Flavor 1 the previous day. This procedure continued for 14 days.

Testing was conducted between grape and cherry saccharin once each day for $\mathbf{8}$ days, as in Test Phase 1 of Experiment 1.

\section{Results}

With the 14 days of training averaged across groups, the rats in Group Half consumed 7.6 and $3.8 \mathrm{ml}$ of Flavors 1 and 2, respectively, and Group Equal drank $6.6 \mathrm{ml}$ of each.

In test, as Figure 5 shows (averaged over days), both groups preferred the flavor of saccharin that followed chocolate milk to the flavor that occurred alone. An ANOVA conducted on test data showed that this difference was significant $[F(1,18)=12.76, p<.01]$. Consumption of flavored saccharin did not vary by group $[F(1,18)=.25, p>.05]$.

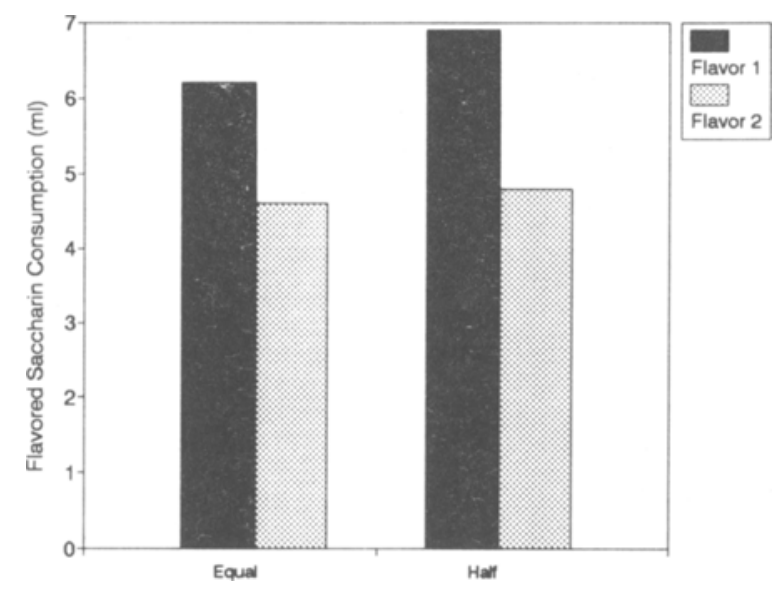

Figure 5. Mean consumption (in milliliters) of the flavor saccharin that followed chocolate milk (Flavor 1) and the flavor that occurred alone (Flavor 2) for each group averaged over testing in Experiment 3. 


\section{Discussion}

In Experiment 3, flavor-nutrient learning was demonstrated again, once exposure to flavored saccharin was controlled; both groups were prevented from drinking more of saccharin Flavor 2 than Flavor 1, and they subsequently preferred Flavor 1 . Because chocolate milk is nutritive, the results of Experiment 3 can be attributed to flavor-nutrient learning. In Experiment 1, which showed no flavor-nutrient learning, the rats consumed more of Flavor 2 in training and did not prefer Flavor 1 in test. In that case, it appears that differential exposure opposed flavor-nutrient learning.

The hypothesis that an unpleasant feeling accompanied the consumption of the flavor of saccharin following chocolate milk was not supported by the results of Experiment 3. Although consumption of the flavor of saccharin that occurred alone was controlled here, consumption of the flavor of saccharin that followed chocolate milk was not; the rats could drink as much of Flavor 1 as they wanted, and then the amount of Flavor 2 was yoked to this consumption. Therefore, the unpleasant state that was theorized to accompany the consumption of saccharin Flavor 1 would still have been present in Experiment 3 and, had it been a factor, would have dictated a preference for Flavor 2-the flavor that occurred alone. However, the test results show that the rats preferred saccharin Flavor 1 to Flavor 2.

The results of Experiment 3 support the hypothesis that the fullness effect controls exposure, and exposure effects can oppose flavor-nutrient learning. Here, we controlled exposure by ensuring that no animal had more experience with Flavor 2 than with Flavor 1; the two were either equal, or twice as much Flavor 1 as Flavor 2 was given. By limiting experience with Flavor 2, exposure could not work against flavor-nutrient learning and, under these conditions, the rats preferred the flavor of saccharin that followed chocolate milk.

An interesting aspect of the exposure phenomenon is that there was no interaction of flavor preference with group in Experiment 3. The group that had twice as much Flavor 1 as Flavor 2 did not show a greater preference for Flavor 1 than the group that had the two flavors in equal amounts. It appears that, although exposure can interfere with flavor-nutrient learning, it does not necessarily enhance it.

\section{GENERAL DISCUSSION}

Three experiments were run to determine the effects of varying exposure to solutions, either by preexposing rats to solutions prior to training (Experiments 1 and 2) or by directly controlling the amount consumed in training (Experiment 3). Although preexposure did not seem to interfere with flavor-nutrient learning, exposure during training was important. Increased consumption of the flavor of saccharin given alone during training opposed flavor-nutrient learning; Experiment 1 showed this. However, increasing consumption of the flavor of saccharin that was paired with calories beyond a level sufficient to produce flavor-nutrient learning did not enhance this learning.

To determine why exposure during training had only a negative effect on flavor-nutrient learning, we consider what is learned in a flavor-nutrient association and in exposure. In flavor-nutrient learning, an association between a flavor and its caloric consequences is learned. Increased consumption through increased exposure, on the other hand, is due to a reduction in neophobia. The rats learn that consuming a particular flavor does not produce illness, and their consumption increases over days until it eventually peaks, as long as there were no ill effects-an increase based on a consequence of no consequence (i.e., no illness). When a procedure is used that allows both flavor-nutrient learning and exposure effects to occur, the one that results in positive consequences (flavor- nutrient learning) may be stronger than the one that is based on no outcome (exposure effects).

There is evidence that animals perform better on operant tasks when the addition of a signal serves as a cue for reinforcement than when the deletion of a signal serves as reinforcement, a phenomenon called the feature positive effect (Hearst \& Wolff, 1989). Something similar to the feature positive effect may have been at work here; the animals demonstrated learning about a positive outcome (a caloric association) over a negative outcome (no illness). By this reasoning, exposure effects may be easily overshadowed by flavor-nutrient learning. However, additional exposure to the flavor paired with calories would not add any more strength to that flavor-calorie association. That would explain why the group in Experiment 3 that had twice as much Flavor 1 as Flavor 2 did not prefer Flavor 1 any more than the group that had equal amounts of saccharin flavors.

The possibility that exposure effects work against flavor-nutrient learning may explain why the results from Capaldi and Sheffer (1992) were different from those in the present Experiment 1. Capaldi and Sheffer's (1992) Experiment 2, in which flavor-nutrient learning was demonstrated with chocolate milk and saccharin, had no preexposure, 38 training days (19 exposures to the pairing of chocolate milk and saccharin Flavor 1 and 19 exposures to only saccharin Flavor 2) and 8 test days. In the present Experiment 1, there were 8 preexposure days, 8 days of training (four exposures to the chocolate milk and saccharin Flavor 1 pairing and four exposures to only saccharin Flavor 2), and 8 days of test. The long training phase with numerous pairings of chocolate milk and saccharin Flavor 1 in Capaldi and Sheffer's Experiment 2 may have been optimal for allowing flavor-nutrient learning to become strong enough to overcome exposure effects. Also, in that experiment, consumption of the two saccharin flavors was about equal by the end of training, whereas in our Experiment 1 more Flavor 2 than Flavor 1 was consumed.

We have shown that rats prefer a flavor that follows a nutrient to a flavor that is given alone. The postinges- 
tive effects of the nutrient are more strongly associated with the flavor that is ingested while those effects are occurring. Compatible results were reported by Capaldi, Campbell, Sheffer, and Bradford (1987), but were termed a dessert effect. In Experiment 4 of that paper, a flavor of saccharin following mash was preferred to a flavor of saccharin given alone. This finding was attributed to a contrast effect. According to this theory, saccharin is a better reward than mash because of its sweet taste. The flavor of saccharin that followed the mash, because it was given in the context of the less preferred mash, was preferred more than a flavor of saccharin given alone. In the present Experiment 2, however, it was shown that contrast did not affect flavor conditioning. The results from the experiments reported here suggest another explanation for Capaldi et al.'s results in terms of flavornutrient learning rather than contrast: The flavor of saccharin that followed the mash was associated with the postingestive effects of the mash, and the preference found in test was due to this association.

Although we have suggested that the preference for the flavor paired with calories here and in Capaldi and Sheffer (1992) represents flavor-nutrient learning, the possibility that the learning is due to a flavor-flavor association should be considered. The flavor of saccharin that followed the chocolate milk may have become associated with the palatable flavor of chocolate milk quite aside from its caloric value. This association might have led to a preference for that flavor in test. A review of flavor-flavor learning will put this possibility into perspective.

Flavor-flavor learning demonstrations are often shown when two flavors are mixed together in the same solution. For instance, Holman (1975) gave rats Flavor 1 in a high concentration of saccharin and Flavor 2 in a low concentration of saccharin, and subsequently tested the two flavors in an equally sweet solution. He found a preference for the flavor that had been in the higher concentration of saccharin. Lavin (1976) conducted a study in which flavors were delivered in succession, and found that, when the flavors were delivered separately, the longest delay at which learning occurred was $9 \mathrm{sec}$. The delay we used between the chocolate milk and the flavored saccharin was $5 \mathrm{~min}$, so it is highly unlikely that flavorflavor learning was responsible for the preference in test. Also, in Lavin's experiment, conditioning to one flavor that preceded another was measured. In the present exper- iments, conditioning to a flavor that followed chocolate milk was measured. If we assume that the flavor of saccharin was associated with the flavor of the preceding chocolate milk, we also assume the possibility of backward conditioning at a fairly long delay. Flavor-nutrient learning does not require postulation of backward conditioning.

The present results suggest that when a flavor follows a nutrient, flavor-nutrient learning is a reliable findingone that has implications for the everyday eating behavior of humans. In consuming a meal and then dessert, one could argue that the calories from the meal would become associated with the flavor of the dessert, thereby increasing a preference for dessert.

\section{REFERENCES}

BOAKES, R. A., \& LUBART, T. (1988). Enhanced preference for a flavour following reversed flavour-glucose pairing. Quarterly Journal of Experimental Psychology, 40B, 49-62.

Capaldi, E. D., Campbell, D. H., Sheffer, J. D., \& Bradford, J. P. (1987). Non-reinforcing effects of giving "dessert" in rats. Appetite, 9. 99-112.

Capaldi, E. D., \& Sheffer, J. D. (1992). Contrast and reinforcement in consumption. Learning \& Motivation, 23, 63-79.

Domjan, M. (1976). Determinants of the enhancement of flavored-water intake by prior exposure. Joumal of Experimental Psychology: Animal Behavior Processes, 2, 17-27.

Domjan, M. (1977). Attenuation and enhancement of neophobia for edible substances. In L. M. Barker, M. R. Best, \& M. Domjan (Eds.), Learning mechanisms in food selection (pp. 151-180). Waco, TX: Baylor University Press.

FlaherTy, C. F. (1982). Incentive contrast: A review of behavioral changes following shifts in reward. Animal Learning \& Behavior, 10, 409-440.

Hearst, E., \& WolfF, W. T. (1989). Addition versus deletion as a signal. Animal Learning \& Behavior, 17, 120-133.

Holder, M. D. (1991). Conditioned preferences for the taste and odor components of flavors: Blocking but not overshadowing. Appetite, 17, $29-45$.

Holman, E. W. (1975). Immediate and delayed reinforcers for flavor preference in rats. Learning \& Motivation, 6, 91-100.

KaMIN, L. J. (1969). Predictability, surprise, attention, and conditioning. In B. A. Campbell \& R. M. Church (Eds.), Punishment and aversive behavior (pp. 279-296). New York: Appleton-Century-Crofts.

Lavin, M. J. (1976). The establishment of flavor-flavor associations using a sensory preconditioning training procedure. Learning \& Motivation, 7, 173-183

(Manuscript received June 1, 1992; revision accepted for publication January 15, 1993.) 